Ann. Zootech., I967, 16 (3), 223-234

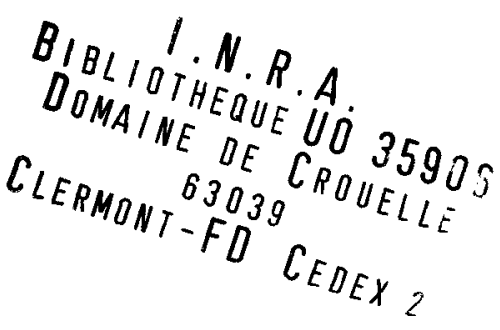

\title{
UTILISATION ÉNERGÉTIQUE, PAR LE RAT EN CROISSANCE, DES PRINCIPALES CÉRÉALES COMPOSANT DES RÉGIMES ISOAZOTÉS ET ÉQUILIBRÉS EN ACIDES AMINÉS
}

\author{
M. VERMOREL et J. KELLER ( $\left.{ }^{1}\right)$ \\ avec la collaboration technique de Jacqueline ThivaL, J. BEJot et R. Manain \\ Station d'Étude des Métabolismes, \\ Centre de Recherches zootechniques et vétérinaires sur les Ruminants, \\ 63 - Theix, près Clermont-Ferrand \\ Institut national de la Recherche agronomique
}

\section{SOMMAIRE}

Des régimes isoazotés et équilibrés en acides aminés, comportant respectivement 85 p. 100 environ de chacune des 4 céréales suivantes : orge, blé, maïs, avoine nue, ont été distribués à des rats en croissance. Dans une première expérience, l'appétibilité comparée et les valeurs de l'énergie métabolisable des céréales ont été déterminées. Dans une seconde expérience, des rats prépubères de même poids ont reçu des quantités voisines d'énergie métabolisable. Les bilans énergétique et carboné ont été obtenus par calorimétrie indirecte. On n'a pas observé de différence, entre les quatre régimes, dans le rendement de l'énergie métabolisable au-dessus de l'entretien. La rétention azotée a été la plus élevée avec les régimes blé et avoine nue. La synthèse de protéines a augmenté avec le bilan énergétique dans le cas du régime "blé " alors qu'elle semble constante avec le régime " maïs". Les résultats sont discutés par rapport aux caractéristiques des céréales

\section{I. ---.- IN'TRODUCTION}

La consommation d'aliments concentrés par le bétail s'accroît continuellement en raison de l'intensification et de l'industrialisation de la production de viande. Les céréales fournissent actuellement les trois quarts de l'énergie et la moitié des protéines consommées; la création de lignées plus riches en matières azotées va encore augmenter cette proportion. Une meilleure connaissance des besoins des animaux et de la valeur nutritive des aliments doit permettre de mettre au point des régimes simplifiés, plus faciles à constituer.

(1) Adresse actuelle. — Zaklad Hodowli Doswiadczalnej Zwierzat, Nowy Swiat 72, Varsovie (Pologne). 
De nombreux travaux ont été consacrés à la détermination de l'énergie métabolisable des céréales, essentiellement sur le Rat, le Poulet et le Porc. Ce critère n'est pas suffisant pour déterminer la valeur énergétique d'un aliment introduit en proportions variables dans un régime complexe. Dès le début du siècle, KELLNER choisit comme critère l'énergie nette d'engraissement. FRAPs (I939) établit la notion d'énergie productive pour caractériser les régimes des poulets en croissance; mais l'énergie productive d'un aliment varie avec le niveau d'alimentation, la composition du régime, l'activité de l'animal, la température (HILI et ANDERson, I958). NeHRING et al. (I963) ont établi, sur cinq espèces animales, (Rat, Lapin, Porc, Ovin, Bovin) les valeurs de l'énergie métabolisable et de l'énergie nette pour l'engraissement de quelques céréales (maïs, orge, avoine), apportées en supplément (30 à $50 \mathrm{p}$. IOO) à une ration de base. Ces déterminations constituent une partie de leurs études générales (par calorimétrie indirecte), destinées à la mise au point d'un système de calcul de l'énergie nette des aliments d'après leur teneur en éléments digestibles.

Particulièrement intéressés par les problèmes concernant la croissance, nous avons été conduits à déterminer la valeur énergétique des céréales comme constituants essentiels de régimes équilibrés en matières azotées et en acides aminés, dans le cas d'une synthèse importante de protéines. Les études ont été effectuées sur Rat en croissance, avec quatre céréales : orge d'automne, blé (Cappelle), maïs hybride, avoine nue (lignée $L \times A 14$ ) ; cette dernière se montre très intéressante en raison de sa teneur en azote, de la composition de ses matières azotées et de sa productivité (PACQUin et al., I965). Par suite de la variabilité individuelle dans la composition des carcasses, nous avons fait appel à la calorimétrie indirecte pour obtenir des bilans énergétiques de courte durée précis au cours de la phase prépubertaire.

Une première expérience (I) a permis de classer les céréales selon leur appétibilité, de déterminer l'énergie métabolisable de chaque régime et d'effectuer quelques bilans azotés. Dans une deuxième expérience (II), nous avons réalisé le bilan énergétique et le bilan carbone-azote (bilan C-N) sur 3 à 5 sujets pour chaque régime.

\section{II. -- MATÉRIEL ET MÉTHODES}

\section{Animaux}

Nous avons utilisé des rats mâles Wistar provenant de l'élevage du Laboratoire, sevrés vers $65 \mathrm{~g}$ (24 jours). L'expérience I a comporté 5 animaux pour chaque régime. Dans l'expérience II, nous avons choisi des sujets âgés de 5 semaines environ et pesant I Io à II $5 \mathrm{~g}$ au moment de leur entrée en chambre respiratoire; cette période correspond à une synthèse importante de protéines corporelles (35 à $55 \mathrm{p}$. Ioo de l'énergie fixée). Une détermination supplémentaire de la digestibilité de chaque régime a été effectuée sur deux animaux pendant 26 jours, de I 5 à $240 \mathrm{~g}$ environ.

\section{Céréales}

Pour des raisons matérielles, des lots de céréales différents ont été étudiés dans les deux expériences. Dans l'expérience II, nous avons choisi des céréales ayant des teneurs voisines en matières azotées totales, sauf pour l'avoine nue qui est beaucoup plus riche (tabl. 3). Leur composition en acides aminés a été estimée d'après celle des céréales de même variété et de teneur azotée voisine (PION et FaUCONNEAU 1966). 


\section{Régimes}

Pour les expériences I et II la composition des régimes est indiquée respectivement dans les tableaux I et 2. La complémentation azotée par la farine de hareng de Norvège et par les acides aminés de synthèse a été calculée d'après les recommandations du X. R. C. (1963); les apports d'acides aminés de synthèse, par kg d'aliment, sont indiqués dans les tableaux i et 2. Dans l'expérience $\mathrm{I}$, les céréales représentaient $8 \mathrm{I}$ à $87 \mathrm{p}$. Ioo de l'énergie brute et fournissaient 50 à $76 \mathrm{p}$. IOO des matières azotées des régimes (tabl. I). Dans l'expérience II, ces proportions étaient de 84 à 88 p. Ioo pour l'énergie brute et de deux tiers environ pour les matières azotées, sauf dans le cas de l'avoine nue (tabl. 2). Les régimes étaient distribués une fois par jour, sous forme de bouillie pour éviter les gaspillages. Les animaux avaient de l'eau à leur disposition, en permanence.

TABLEAU I

Eixpérience I

Composition et caractéristiques des régimes, principaux résultals

\begin{tabular}{|c|c|c|c|}
\hline & Orge & Blé & Maïs \\
\hline \multicolumn{4}{|l|}{ Composition des régimes (g de M. S.) } \\
\hline Céréale $\ldots \ldots \ldots \ldots \ldots \ldots \ldots \ldots \ldots$ & 781,6 & 832,0 & 748,4 \\
\hline Farine de hareng de Norvège ........... & 75,0 & 31,7 & 86,5 \\
\hline Acides aminés de synthèse $\ldots . . . \ldots \ldots \ldots$ & 7,250 & 10,800 & 7,600 \\
\hline Agar-agar $\ldots \ldots \ldots \ldots \ldots \ldots \ldots \ldots$ & 20 & 20 & 20 \\
\hline Mélange vitaminique + choline...$\ldots \ldots \ldots$ & 10 & 10 & 10 \\
\hline Huile de Mais (+ Vit. A et $D) \ldots$. & 20 & 20 & 20 \\
\hline Mélange minéral de OsBORNE et MendeL ... & 20 & 20 & 20 \\
\hline Alunozal $\ldots \ldots \ldots \ldots \ldots \ldots \ldots \ldots \ldots$ & 0,4 & 0,5 & 0,2 \\
\hline \multicolumn{4}{|l|}{ Supplément d'acides aminés de synthèse $(\mathrm{g})$} \\
\hline$d l$-thréonine $\ldots \ldots \ldots \ldots \ldots \ldots \ldots$ & 1,800 & 3,500 & 1,640 \\
\hline 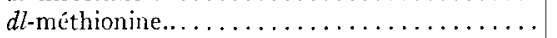 & 0,450 & 0,500 & 0,750 \\
\hline 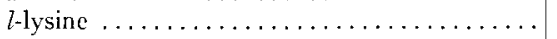 & 2,900 & 5,300 & 3,200 \\
\hline$l$-histidine $\ldots \ldots \ldots \ldots \ldots \ldots \ldots \ldots$ & 1,000 & 0,900 & 0,700 \\
\hline$l$-tryptophane $\ldots \ldots \ldots \ldots \ldots \ldots \ldots \ldots$ & 1,100 & 0,600 & 1,350 \\
\hline \multicolumn{4}{|l|}{ Caractérisliques des régines } \\
\hline Cúrógle / Énergie brute.... . | & 82,6 & 87,5 & 81,1 \\
\hline Céréale I) 100 régime Azote ......... & 54,2 & 76,3 & 50,0 \\
\hline Energic brute moyenne (kcal/g iI. S.) ..... & 1,393 & 4,381 & 4,558 \\
\hline$N \times 6,25$ p. 100 M..$\ldots \ldots \ldots \ldots \ldots \ldots$ & 15,1 & 15,2 & 15,2 \\
\hline $\mathrm{CUD}_{a}$ énergie $\ldots \ldots \ldots \ldots \ldots \ldots \ldots \ldots$ & $80,3 \pm 0,2$ & $86,0 \pm 0,2$ & $87,9 \pm 0,4$ \\
\hline$N$ digestible $(\mathrm{mg}) /$ Fnergie mútabolisable (kcal) & 5,25 & 5,23 & 1,90 \\
\hline \multicolumn{4}{|l|}{ Résullals } \\
\hline Energie métabolisable / kcal/g X. S....... & 3,525 & 3,800 & 4,080 \\
\hline de la céréale $\{$ p. 100 de l'orge.... & 100 & 108 & 116 \\
\hline $\begin{array}{c}\text { Gain de poids vif moyen (g/jour) (j sujets } \\
\text { pendlant } \delta \text { semaines } \ldots \ldots \ldots \ldots \ldots \ldots \ldots \ldots\end{array}$ & 4,78 & 5,25 & 4,10 \\
\hline $\begin{array}{l}\text { Matière sèche ingèré }(\mathrm{g} / \text { jour) (3 sujets pen- } \\
\text { dant } 5 \text { semaines) } \ldots \ldots \ldots \ldots \ldots \ldots \ldots \ldots\end{array}$ & 16,0 & 17,5 & $15, / \mathrm{k}$ \\
\hline Énergie digestible ingérée (kcal/jour) (3 sujets & & & \\
\hline pendant 5 semaines)..$\ldots \ldots \ldots \ldots \ldots$ & 56,2 & 65,9 & 61,2 \\
\hline Gain de poids vif (g)/Azote ingéré $(g) \ldots \ldots$ & $13,2+0,2$ & $14,5 \doteq 0,5$ & $11,+11,5$ \\
\hline Coefficient de rétention azotée apparent .... & $49,0=1,9$ & $49,9 \doteq 1,1$ & \\
\hline
\end{tabular}


Tous les sujets ont reçu les régimes expérimentaux dès le sevrage. Dans l'expérience I, tous les régimes ont été distribués ad libitum. Dans l'expérience II, seul le régime "Maïs " a été offert ad libitum ; pour les autres régimes, les distributions ont été limitées de façon à ajuster les quantités d'énergie métabolisable ingérées. Les quantités distribuées et les refus ont été pesés respectivement à l'état frais et après séchage à l'étuve ( 24 heures à $\left.70^{\circ} \mathrm{C}\right)$.

Les fèces des animaux recevant les régimes "Orge ", "Blé ", "Maïs " étaient d'une consistance molle ou fluide ; on y a remédié en ajoutant 0,2 à $0,5 \mathrm{~g}$ d'alunozal par $\mathrm{kg}$ d'aliment.

\section{TABLEAU 2}

Expérience II

Composition et caractéristiques des régimes, principaux résultats

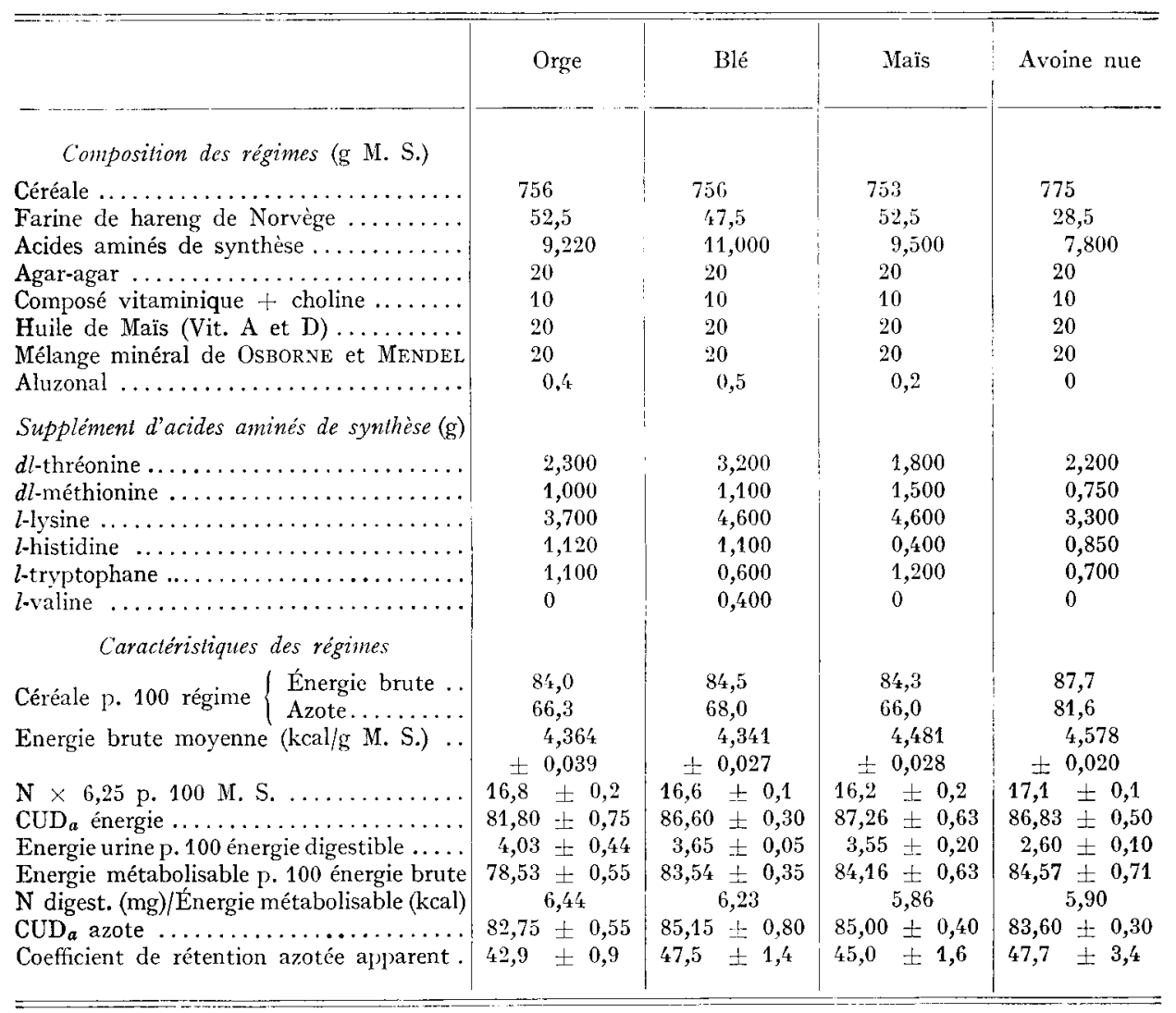

\section{Calorimétrie indirecte}

Les échanges gazeux respiratoires ont été mesurés au moyen d'une installation du type "Circuit Fermé "; le gaz carbonique produit est déterminé gravimétriquement après absorption par des tamis moléculaires et l'oxygène est fourni par un spiromètre. Les animaux ont été maintenus à une température de $22 \pm 0,5^{\circ} \mathrm{C}$. (La description complète de l'installation et des méthodes sera exposée dans une autre publication) (VERMOREL, I967).

Pour chaque sujet, une période d'adaptation de 3 à 5 jours était prévue dans une cage à métabolisme et dans une chambre respiratoire identiques à celles utilisées pendant l'expérience. Les mesures des échanges respiratoires ont été effectuées pour chaque rat, pendant une période de 9 jours consécutifs, avec des interruptions de Io minutes par jour. 


\section{Analyses}

Chaque jour, un échantillon représentatif de l'air de la chambre respiratoire était prélevé en début de mesure et deux échantillons en fin de mesure ; la composition de l'air a été déterminée avec l'appareil de CaRPEnTER et HALDANE.

Deux échantillons de régime frais, prélevés quotidiennement au moment de la distribution des régimes, ont été séchés à l'étuve et conservés pour les analyses par périodes de 4,5 ou 9 jours. Les fèces, conservées à - ${ }_{15}^{\circ} \mathrm{C}$, ont été lyophilisées. L'urine a été lyophilisée en sachets de polythène (NiJKaMP, Ig64).

L'énergie brute des ingesta et des excreta a été déterminée dans un calorimètre semi-adiabatique ; l'azote a été dosé par la méthode macrokjeldahl et le carbone par la méthode de Dumas.

\section{RÉSULTATS}

\section{Expérience I}

L’appétibilité des régimes a été estimée d’après les gains de poids vif des rats (5 animaux par lot) au cours d'une période de 5 semaines et d'après leurs consommations ( 3 animaux par lot (tabl. I). Les gains de poids vif moyens ont été les suivants pour les trois régimes : "Blé " 5,25 g, "Orge " 4,78 g, "Maïs » 4, Io g par jour. Les quantités de matière sèche et d'énergie digestible ingérées ont été les plus élevées avec le régime "Blé " avec le régime "Maïs ", la faible consommation de matière sèche a été partiellement compensée par la digestibilité élevée de l'énergie. Dans l'expérience II, le comportement alimentaire des animaux a confirmé les premiers résultats ; pour cette raison, on peut considérer que 1'appétibilité de l'avoine nue est voisine de celle du blé.

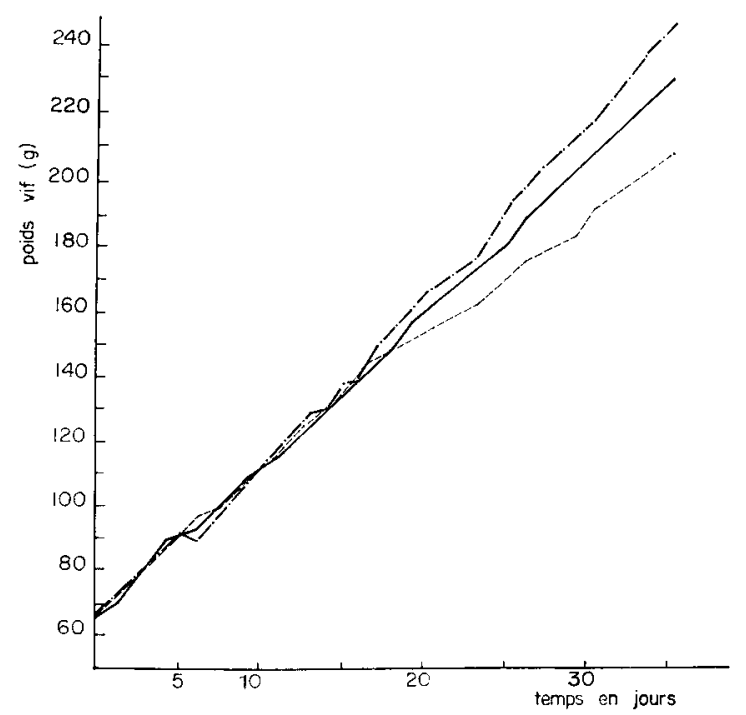

FIG. I. - Gains de poids vif moyens des rats (5 sujets par lot) après le sevrage, avec les 3 régimes

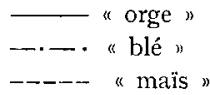


Les coefficients d'utilisation digestive apparents (CUD $)$ del'énergie, déterminés sur 3 animaux pendant 4 semaines, ont été les suivants, pour les régimes" Orge ", "Blé " et "Maïs " 80,3 - 86,0 - 87,9. Correction faite pour les compléments, les valeurs de l'énergie métabolisable des céréales rapportées à celle de 1'orge ont été les suivantes, respectivement : IOO, IO8, II6. L,es valeurs du coefficient d'efficacité protéique, du coefficient de rétention azotée apparent $\left(\mathrm{CR}_{\alpha}=\frac{\mathrm{N} \text { retenu }}{\mathrm{N} \text { ingéré }}\right)$ sont indiquées dans le tableau $\mathrm{I}$.

\section{Expérience $I I$}

Les $\mathrm{r}_{4}$ animaux pesaient II2,5 $\pm 3 \mathrm{~g}$ au début et $\mathrm{I}_{54,2} \pm 6 \mathrm{~g}$ à $1 \mathrm{a}$ fin de la période de mesure de 9 jours en chambre respiratoire ; cette croissance correspond à un gain de poids vif moyen de $4,63 \mathrm{~g} /$ jour. Les quantités d'énergie métabolisable consommées ont varié de 46,4 à $52,8 \mathrm{kcal} /$ jour. Pour une même consommation d'énergie métabolisable (5o kcal/jour), les gains de poids vif moyens ont été les suivants : régimes "Blé » et "Orge " 4,8 g/jour, "Avoine nue " 4,5 g/jour " Maîs " 4,2 g/jour.

TABI.EAU 3

Caractéristiques des céréales étudièes dans l'expérience II

\begin{tabular}{|c|c|c|c|c|}
\hline & Orge & Blé & Maïs & Avoine nue \\
\hline 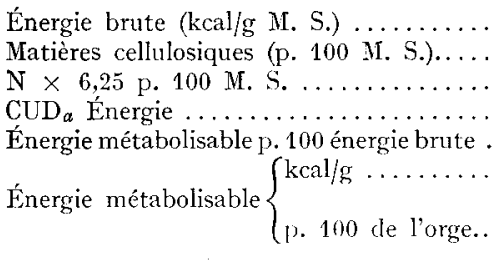 & $\begin{array}{c}1,130 \\
6,05 \\
12,57 \\
81,8 \pm 0,8 \\
79,2 \pm 0,6 \\
3,508 \pm 0,027 \\
100\end{array}$ & $\begin{array}{c}4,410 \\
2,52 \\
12,92 \\
87,5 \pm 0,3 \\
85,0 \pm 0,4 \\
3,749 \\
\pm 0,018 \\
107 \pm 1,2\end{array}$ & $\begin{array}{c}1,545 \\
2,57 \\
12,53 \\
88,2 \pm 0,6 \\
85,8 \pm 0,6 \\
3,899 \\
\pm 0,028 \\
111 \pm 1,2\end{array}$ & $\begin{array}{c}4,660 \\
1,58 \\
14,56 \\
87,8 \pm 0,5 \\
85,8 \pm 0,7 \\
3,997 \\
\pm 0,033 \\
11^{\prime} \pm 1,3\end{array}$ \\
\hline
\end{tabular}

L,es valeurs des CUD $a$ de l'énergie et de 1'azote, de l'énergie métabolisable et des coefficients de rétention azotée sont groupées dans les tableaux 2 et 3. La valeur du CUD $a$ de l'énergie est la moyenne des 3 à 5 valeurs obtenues sur 9 jours et des deux valeurs obtenues sur 26 jours. En général, le $\mathrm{CUD}_{a}$ de l'énergie a été plus élevé pour la période de 26 jours que pour la période de 9 jours; les différences "ont été : 0,9 pour le régime "Orge", zéro pour le "Blé ", I,I pour le "Maïs et 0,85 pour 1' "Avoine nue ». Le même phénomène a été observé dans l'expérience I.

Les CUD $a$ de 1'énergie des régimes "Blé " et "Avoine nue " sont semblables $(86,60$ et 86,83$)$ et légèrement inférieurs à celui du "Maïs " $(87,26)$. L'énergie perdue dans 1'urine étant plus faible pour le régime "Avoine nue ", le pourcentage d'énergie métabolisable dans l'énergie brute est un peu plus élevé pour " l'Avoine nue " $(84,57)$ que pour le "Maïs " $(84, \mathrm{I} 6)$ et le "Blé " $(83,54)$.

Les CUD $a$ de l'énergie des céréales ont été calculés à partir de ceux des régimes en appliquant aux compléments les $\mathrm{CUD}_{a}$ suivants : farine de poisson et acides aminés de synthèse 9o, amidon 95, huile de maïs 98, agar-agar zéro. 
Nous avons fait l'hypothèse qu'il n'y a pas de différence importante dans l'utilisation des matières azotées puisque les régimes sont équilibrés en acides aminés ; nous avons calculé la part de 1'énergie de 1'urine provenant de la céréale d'après le pourcentage de matières azotées apporté par cette céréale, Forbes (1938) ayant montré que 1'énergie de l'urine est proportionnelle à l'azote excrété. Les proportions d'azote et d'énergie apportées par les céréales étant voisines, les $\mathrm{CR}_{a}$ peul différents et l'énergie de l'urine ne représentant que 2,6 à 4,0 p. roo de l'énergie digestible des régimes, l'erreur commise dans l'estimation de l'énergie métabolisable est négligeable pour la comparaison des quatre céréales. L'énergie métabolisable de chaque céréale a été obtenue en multipliant le $\mathrm{CUD}_{a}$ de l'énergie par le pourcentage d'énergie métabolisable dans l'énergie digestible (tabl. 3).

Les différences entre le bilan calorique et le bilan $\mathrm{C}-\mathrm{N}$ ont été de $\pm 0,9 \mathrm{p}$. Ioo de l'énergie ingérée et $\pm 3,8 \mathrm{p}$. Ioo du bilan énergétique. Le bilan $\mathrm{C}-\mathrm{N}$ a été généralement supérieur at bilan énergétique en raison des pertes probables de carbone au cours de la lyophilisation de l'urine. Nous n'avons conservé que les valeurs du bilan énergétique pour la comparaison des résultats.

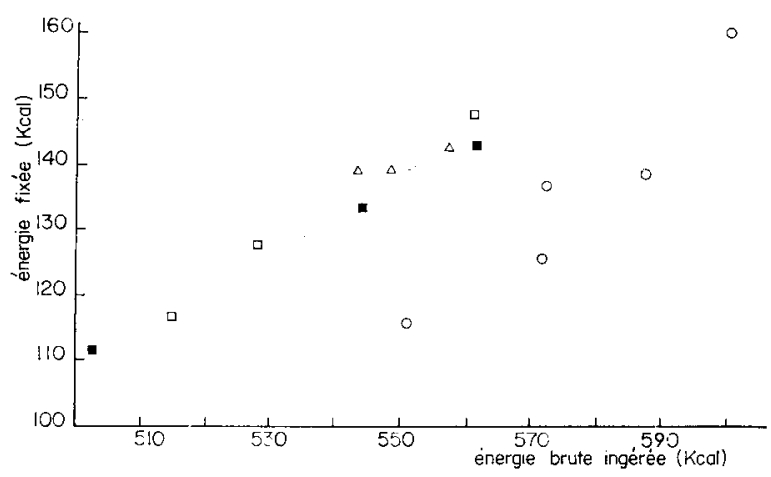

FIG. 2. - Relalion enire l'énergie brule ingérée el l'énergie flxée, pendanl une période de 9 jours avec les 4 régimes:

$$
\begin{aligned}
& \text { - "orge" } \\
& \text { " blé " } \\
& \square \text { " maïs" } \\
& \Delta \text { "avoine nue " }
\end{aligned}
$$

La figure 2 représente les quantités d'énergie fixée en fonction de l'énergie brute ingérée pour les 4 régimes. Dans la figure 3 nous avons regroupé les valeurs de l'énergie fixée en fonction de l'énergie métabolisable ingérée ; il n'y a pas de différence apparente entre les régimes et les points se répartissent de part et d'autre d'une droite de régression dont l'équation est :

$$
\mathrm{Y}=0,706 x-\mathrm{r} 85,5
$$

Cette relation montre que pour de telles croissances, le rendement de l'énergie métabolisable au-dessus de l'entretien est semblable pour les quatre régimes et égal à 70,6 p. IOo. Le besoin d'entretien correspond à la quantité d'énergie métaboli- 
sable qui assure un bilan énergétique nul; on peut l'estimer à $262,8 \mathrm{kcal}$ pour 9 jours, soit 29,2 kcal d'énergie métabolisable par jour, pour un rat d'un poids vif moyen de $\mathrm{I} 33,4 \mathrm{~g}$.

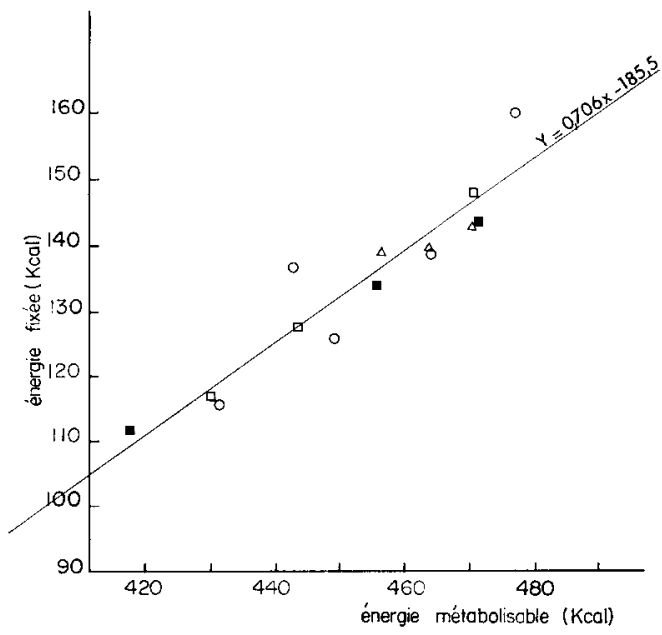

FIG. 3. - Relation entre l'énergie métabolisable ingérée et l'énergie fixée, pendant une période de 9 jours avec les 4 régimes:

$\circ$ " orge"

- "blé "

ㅁ mais"

$\Delta$ " avoine nue "

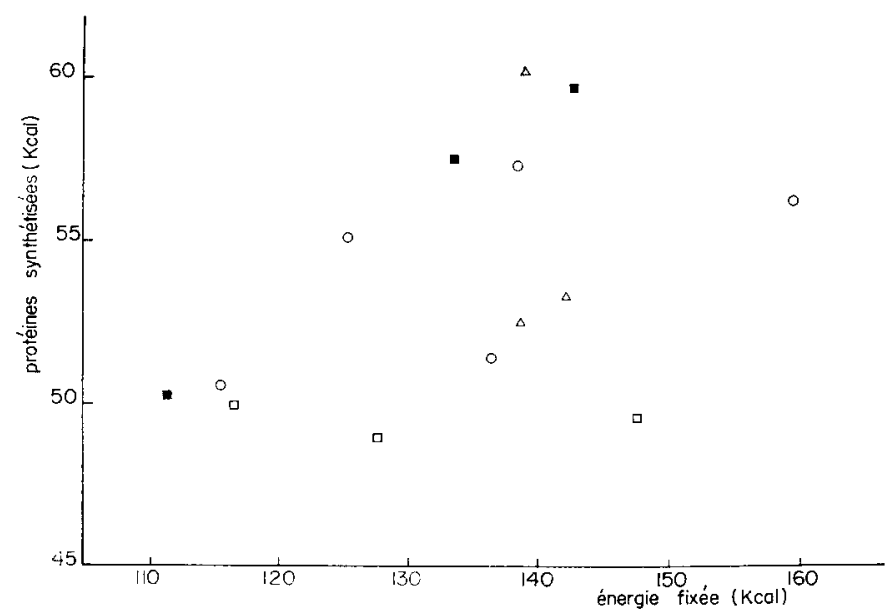

FIG. 4. - Relation entre l'énergie fuxée (kcal) et la synthèse de protéines (kcal) pendant une période de 9 jours anec les 4 régimes:

$$
\begin{aligned}
& 0 \text { "orge " } \\
& \text { "blé " } \\
& \square \text { "maïs" } \\
& \Delta \text { "avoine nue " }
\end{aligned}
$$


Les coefficients de rétention azotée apparents des quatre régimes (tabl. 2) montrent que l'azote des régimes "Blé " et "Avoine nue " a été fixé en plus grande proportion que celui de 1' "Orge " et du " Maïs ". Malgré une certaine variabilité des résultats correspondant à un même régime, la figure 4, représentant les quantités d'énergie fixée sous forme de protéines en fonction de l'énergie fixée totale, met en évidence l'augmentation de la synthèse des protéines dans le cas du régime "Blé " et sa constance relative pour le régime "Maïs", quand les bilans énergétiques sont supérieurs à i Io kcal environ.

\section{DISCUSSION}

Nous avons volontairement utilisé des rats avant la puberté présentant une croissance élevée et une synthèse de protéines importante (35 à $44 \mathrm{p}$. Ioo de l'énergie fixée) et d'autre part des régimes équilibrés en matières azotées comportant $85 \mathrm{p}$. Ioo de céréales environ.

\section{TABIEAU 4}

Valeurs relatives de l'énergie métabolisable des céríales (comparées à celle de l'orge) obtenues par différents auteurs sur rats et porcs.

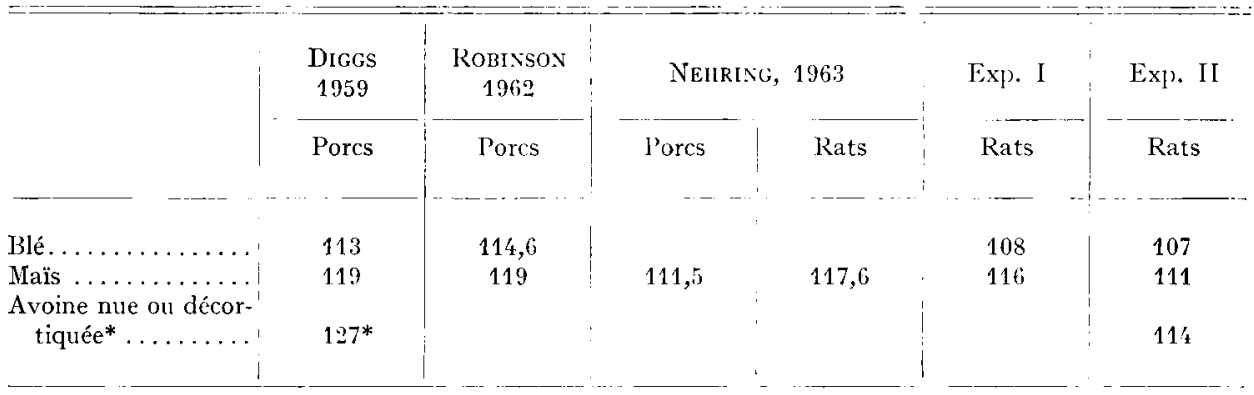

* Thomke (1960) a trouvé la valeur suivante pour l'avoine décortiquée sur porcs : f,140 kcal d'énergie métabolisable par g de matière sèche, c'est-à-dire 103,5 P. 100 de la valeur que nous avons trouvée sur rats.

Le classement des céréales selon leur appétibilité correspond à celui établi par SAIMON-LEGAGNEUR et FÉVRIER (I959) chez le porcelet recevant des aliments granulés. Par opposition au système de supplémentation utilisé par NeHring et al. (I963), le système de substitution d'une céréale à une autre ne permet pas de distinguer l'énergie et l'azote métaboliques fécaux de l'indigestible réel du régime; il nous a permis, en revanche, d'introduire une proportion élevée de céréale dans le régime et d'obtenir les bilans énergétique, carboné et azoté par une seule mesure, ce qui assure une précision supérieure. Nous avons groupé dans le tableau 4 les valeurs relatives de l'énergie métabolisable de ces céréales obtenues par plusieurs auteurs sur Rat et sur Porc. Les différences d'énergie métabolisable des céréales des deux lots sont dues aux variations de leur teneur en lipides et en matières cellulosiques; 
l'écart entre les céréales est plus faible dans l'expérience II que dans l'expérience I par suite de la digestibilité plus élevée de l'énergie de l'orge.

Le faible nombre de mesures effectuées sur chaque régime ne nous permet pas de mettre en évidence des différences éventuelles de rendement de 1'énergie métabolisable entre les céréales. Le rendement de 70,6 p. Ioo est inférieur à ceux observés par NeHRing ( I 963) sur Rat quasi adulte, avec le maïs $(77,3 \pm 2,4)$ et 1'orge $(74,8$ $\pm \mathrm{I}, 7)$ mais il est égal à celui observé avec l'avoine $(70,8 \pm 2, \mathrm{I})$. Ce rendement est supérieur à celui déterminé par Fraps (I939) sur Poulet en croissance avec des régimes complets $(67,6)$ et très supérieur à celui établi par Btis (I96I) sur Rat en croissance $(44,4 \pm 9,8)$ entre 80 et $200 \mathrm{~g}$.

Le besoin d'entretien des animaux intervient dans le calcul de ce rendement; si le besoin d'entretien est surestimé, le rendement est trop élevé. La détermination indirecte de ce besoin $(262,8 \mathrm{kcal}$ d'énergie métabolisable pour 9 jours chez un rat de $133,4 \mathrm{~g})$ est inférieure à la valeur $(288,5 \mathrm{kcal})$ mesurée directement par BFIs (I96I) et aux standards de Brody ( 1945$):(283,5 \mathrm{kcal})$. La détermination directe du besoin d'entretien des animaux en croissance donne des résultats discutables du fait que, d'une part les animaux affamés sont agités, et que d'autre part l'organisme est le siège de réactions de catabolisme et d'anabolisme mal couplées qui augmentent la dépense calorique.

Déduction faite de l'entretien, nous n'avons pas observé de variation du rendement de l'énergie métabolisable avec l'augmentation du niveau d'alimentation ; les quantités d'énergie métabolisable ingérée ont peut-être été insuffisantes, ou bien, les sujets qui ingéraient le plus et tendaient à synthétiser davantage de lipides étaient plus calmes et réduisaient leur besoin d'entretien ; cette hypothèse pourrait expliquer certains bilans énergétiques élevés obtenus avec le régime "Orge ». Nous disposons actuellement d'une deuxième chambre respiratoire qui nous permettra de doubler le nombre de mesures et d'un appareil pour l'enregistrement de l'activité de l'animal.

Le rendement de l'énergie métabolisable au-dessus de l'entretien étant le même pour les quatre régimes, les quantités d'énergie fixée sont voisines et de l'ordre de I32 kcal pour une même ingestion de $45^{\circ} \mathrm{kcal}$ d'énergie métabolisable pendant les 9 jours. Les différences de gains de poids vif des animaux entre les quatre régimes pourraient s'expliquer en partie par la formation de quantités différentes de protéines et de lipides dont rendent compte, dans le cas du blé et du maïs, les C $\mathrm{R}_{a}$ (tabl. 2) et les proportions d'énergie fixée sous forme de protéines (fig. 4).

Les acides aminés indispensables du régime couvrant les besoins d'entretien et de croissance des animaux et l'apport d'acides aminés non indispensables étant excédentaire, un faible rapport Azote digestible/Énergie métabolisable tend à augmenter le $\mathrm{CR}_{a}$, ce qui est le cas pour les régimes " Maïs " et "Avoine nue ». La valeur élevée du $\mathrm{CR}_{a}$ observée avec le régime "Avoine nue " est en accord avec les résultats de CARPENTER et CIEGG (I958) sur Poulet; compte tenu de la valeur énergétique nette également élevée, elle montre la bonne valeur nutritive de cette céréale. La rétention azotée importante observée avec le régime "Blé "s'explique à la fois par la digestion rapide de cette céréale et par 1'absorption simultanée des acides aminés des différentes sources azotées du régime, blé, farine de poisson, acides aminés “ de synthèse (PION et RERAT, I967).

L'absorption non simultanée des acides aminés des trois sources, due à la digestion lente de l'orge (PION et al., I964), pourrait expliquer la faible rétention azotée 
avec ce régime : le pool sanguin et musculaire des acides aminés libres indispensables peut assurer les besoins d'anabolisme chez le Rat en croissance, pour une durée de 1'ordre d'une demi-heure (PAWLAK et Pron, I967).

Dans le cas du régime "Maïs ", l'excès de leucine (leucine/isoleucine $=2,80$ ), compte tent de la faible proportion (50 p. Ioo) d'acides aminés non indispensables et du niveau azoté peu élevé du régime, pourrait être à l'origine de la faible rétention azotée (SPOLTER et HARPER, I96I) ; la disponibilité réduite de certains acides aminés peut aussi être en cause. La synthèse réduite de protéines pourrait expliquer en partie la faible appétibilité de cette céréale.

Du point de vue de l'énergie nette, l'avoine nue et le maiss sont les plus riches, en raison de la teneur élevée en amidon et en lipides du mais et de la teneur élevée en lipides de l'avoine nue; le blé a une valeur un peu plus faible et 1'orge est plus pauvre par suite de sa teneur élevée en matières cellulosiques et en hémicelluloses. Si on s'intéresse à la valeur alimentaire des céréales pour les monogastriques en croissance intensive, chez lesquels l'appétit est souvent un facteur limitant, il faut considérer à la fois l'appétibilité, les valeurs énergétique et azotée. L'avoine nue et les blés les plus riches en azote ( 5 p. Ioo de matières azotées par rapport à la matière sèche), moyennant une légère supplémentation par des acides aminés de synthèse (lysine, tryptophane), semblent pouvoir permettre une croissance élevée chez les rats (PAwLAK, I967) ; les blés normaux doivent être supplémentés par un faible apport de protéine de bonne qualité, et d'acides aminés de synthèse. Le mais et l'orge doivent être introduits dans des régimes plus complexes comportant d'autres céréales ou des sources de protéines complémentaires.

Reçu pour publication en avril 1967.

\author{
SUMMARY
}

THE UTILIZATION OF THE ENERGY OF FOOD BY THE GROWING RAT : ENERGETIC VALUE OF CEREAL DIETS OF EQUAL NITROGEN LEVELS AND ADJUSTED FOR AMINO-ACIDS

Diets of equal nitrogen levels and adjusted for amino acids according to growth requirements were given to growing rats. About 85 per cent of each diet was one of the following main cereals : barley, wheat, maize, naked oat. The four cereals were supplemented with herring meal and synthetic amino-acids (tables $I$ and 2). In a first experiment the palatability, digestibility, metabolizable energy and nitrogen retention (table 2) of the cereals were determined.

In a second experiment, rats weighing I 10 to I $5 \mathrm{~g}$ were given similar amounts of metabolizable energy ; their average growth rate was $4.6 \mathrm{~g}$ per day. Energy and carbon balances were obtained by meant of indirect calorimetry in a closed-circuit apparatus, for 9 days running periods (fig. 2). Tables 2 and 3 show the characteristics of diets and cereals. The values of metabolizable energy of ccreals referred to barley (IOO) are 107 , III, II4 respectively for wheat, maize and naked oat. No difference in metabolizable energy efficiencies was noticed between the 4 diets (fig. 3 ) and the mean efficiency was 70.6 per cent. Maintenance needs may be estimated to $29.4 \mathrm{Kcal}$ metabolizable energy per day with rats weighing $\mathrm{I} 33.4 \mathrm{~g}$ on the average. These results are compared to the literature.

Nitrogen retention was higher with wheat and naked oat diets (table 2). Figure 4 shows that protein synthesis increased in relation to retained energy with wheat, though it seemed constant with maize. The quick digestion of wheat and simultaneous absorption of amino-acids could explain the high nitrogen retention, while barley induces the opposite. With a maize dict, leucine excess (leucine/isoleucine $=2.8$ ) could explain, owing to the low proportion (5० per cent) of dispensable amino-acids and to the low nitrogen content of the diet, the low nitrogen retention. 


\section{RÉFÉRENCES BIBLIOGRAPHIQUES}

Bein L., Igor. N-Bilanz und gesamtstoffwechselversuche an wachsenden Albinoratten. Arch. Tierernähr, 11, 42-70.

Blaxter K. L., Clapperton J. L., Wainman F. W., ig66. Utilization of the energy and protein of the same diet by cattle of different ages. J. Agric. Sci., 67, 67-75.

Brody S., 1945. Bioenergetics and Growth. 478 . Reinhold Publishing Corporation, 33o West Forty. Second Street, New York, U. S. A.

Carpenter K. J., ClegG K. M. I957. The relative value of three cereals as protein sources for growing chicken, Brit. J. Nutr., 11, 358-364.

Diggs B. G., Becker D. E., Terrill S. W., Jensen A. H., I959. The energy value of various feedstuffs for the young pig. J. Anim. Sci., 18, 1492-1493.

FAUCONnEAU G. 1966. Quelques aspects actuels de la nutrition azotée des animaux monogastriques. $I X^{\mathrm{e}}$ Congr. Internat. Prod. Anim. Lidimbourg, août 1966.

Forbes E. B., Leroy-Voris J. W., Bratzler J. W., Walter-Waino I938. The utilization of energy producing nutriment and protein as affected by the plane of protein intake. $J$. Nutr., 15, 285-307.

Fraps G. S., Carlyle E. C., I939. The utilization of the energy of feed by growing chickens. Texas Agric. Exp. Stat. Bull. $57 \mathrm{I}, \mathbf{1}-44$.

Hill F. W., Anderson D. L., 1958. Comparison on metabolizable energy and productive energy determinations with growing chicks. J. Nutr., 64, 587-603.

KIELANOWSKI J., I 964 . Estimates of the energy cost of protein deposition in growing animals. $I I I^{\text {e }} S y m p$. Métab. Energ., Troon. mai 1964 .

Mathieu C. M., Wegat-Litre E., ig60. Les préférences alimentaires du veau. I. Appétibilité comparée des céréales. Ann. Zootech., 9, 262-270.

Milne F. N. J., 1954. The value of maïze in poultry feeding (in Nutr. Abstr. Rev., $1955 \mathrm{n}^{\circ} 3060$ ).

Morimoto H., Yoshida M., Hosini H., rg63. Nutritive value of barley for poultry feed. i. Comparison of nutritive value of eight cereals (in Nutr. Abstr. Rev., I $964 \mathrm{n}^{\circ}$ г $56 \mathrm{I}$ ).

Nehring K., Schiemann R., Hoffmann L., Jentscin W., ig63. Die energetische Verwertung der Futterstoffe. Arch. Tierernähr., 13, I I9-2 I3.

Nijkamp H. J., I964. Some remarks about the determination of the heat of combustion and the carbon content of urine. III $I^{\mathrm{e}}$ Symp. Mélab. Energ. Troon. mai i 964 .

Pacquin J., Moule C., Amice M., i 965 . Influence de l'hybride d'avoine nue $(L \times A)$ I4 sur la croissance des poulets de chair. Bull. Inform. Stat. Ploufragran, 26, I I-I 4 .

PAWLAK M., communications personnelles.

PAWLAK M., Pron R., I967. Influence de la composition des protéines alimentaires sur les teneurs en acides aminés libres du sang total et du muscle du Rat en croissance. C. R. Acad. Sci. (sous presse).

Pion R., Fauconneau G., Rérat A., ig64. Variation de la composition en acides aminés du sang porte au cours de la digestion chez le porc. Ann. Biol. anim., Bioch., Biophys., 4, 383-40r.

Pion R., Fauconneau G., 1966. Les acides aminés des protéines alimentaires. Méthodes de dosage et résultats obtenus. Cahiers de l'A. E. C., 6, I 59 -I 75 .

PION R., RÉRAT A., 1967. Influence d'une supplémentation en lysine sur l'évolution d'aminoacidémie porte du Porc en croissance au cours de la digestion d'une ration à base de blé. C.R. Acad. Sci. (sous presse).

Robinson D. W., Lewis D., ig62. Digestible energy of cereals to pigs. Proc. Nut.Soc., 21, XXVI-XXVII.

Salmon-Legagneur E., FÉvrier R., I959. Les préférences alimentaires des porcelets III. Appétence de quelques céréales. Ann. Zootech, 8, I39-146.

Sauberlich H. E., Chang W. Y., Salmon W. D., I953. The comparative value of corn on high and low protein content for growth in the rat and chick. J. Nutr., 51, 623-635.

Spolter P. D., Harper A. E., I96I. Leucine-isoleucine antagonism in the rat. Amer. J. Physiol., 200, 513-518.

Thомkes., 1960. Studies on the digestibility of oats by pigs. Kungl. Lantbrukshögsk. Ann., 26, 269-288 